Bio - grafía. Escritos sobre la Biología y su Enseñanza. ISSN 2027-1034

Edición Extraordinaria. p.p. 492 - 501

Memorias del IX Encuentro Nacional de Experiencias en Enseñanza de la Biología y la Educación Ambiental. IV Congreso Nacional de Investigación en Enseñanza de la Biología.

\title{
IDENTIFICACIÓN DE ESQUEMAS ARGUMENT ATIVOS A TRAVÉS DE UN CASO SOCIOCIENTÍFICO, EN ESTUDIANTES DE GRADO 8
}

\section{IDENTIFICATION OF ARGUMENTATINES SCHEMES ALTHOUTHG A SOCIO - CIENTIFIC CASE, IN STUDENTS OF GRADE 8}

\author{
Rodríguez Mayorga, Ingrid Bibiana ${ }^{1}$ \\ Suarez, Oscar Jardey ${ }^{2}$
}

\section{RESUMEN}

El objetivo de esta investigación, en desarrollo, es identificar y analizar los esquemas argumentativos de los niños y niñas de grado octavo, de un colegio público de Bogotá Colombia, frente a una situación socio- científica denominada "adicción a sustancias psicoactivas". El estudio se realiza a partir de la implementación de una secuencia didáctica que incluye situaciones elicitadoras así como espacios educativos que motivan la argumentación. El registro de la información, así como la extracción de los datos, se realiza con grabaciones de audio y vídeo de algunas sesiones así como observaciones documentadas en diario de campo. Para realizar la caracterización de la argumentación el estudio se fundamenta en la teoría de Toulmin. Metodológicamente se inicia con una prueba piloto a partir de la situación elicitadora basada en el contexto socio-científico denominado "relaciones sexuales a temprana edad". Los resultados muestran esquemas de argumentación, generalmente incompletos, comunes que no se apoyan en conocimientos esperados. Las reflexiones finales llevan a considerar que se deben hacer esfuerzos por diseñar ambientes de aprendizaje que susciten la argumentación y por ende la toma de decisiones en contexto; en ese sentido la investigación continuará proyectada al caso central: "adicción a sustancias psicoactivas".

PALABRAS CLAVE: argumentación, caso socio - científico, sustancias psicoactivas, toma de decisiones.

\section{ABSTRACT:}

The objective this research, in development, is to identify and to analyze the argumentative schemes of eighth grade children from a public school in Bogotá Colombia, facing a socio - scientific situation called "addiction to psychoactive substances". The study is carried out from the implementation of a didactic sequence that

\footnotetext{
${ }^{1}$ Fundación Universidad Autónoma de Colombia. Maestría en Edumática. Grupo de investigación ciencias básicas, matemáticas y su didáctica. irodriguez.mayorga@fuac.edu.co.

${ }^{2}$ Fundación Universidad Autónoma de Colombia. Maestría en Edumática. Grupo de investigación ciencias básicas, matemáticas y su didáctica. oscar.suarez@fuac.edu.co.
} 
Bio - grafía. Escritos sobre la Biología y su Enseñanza. ISSN 2027-1034

Edición Extraordinaria. p.p. 492 - 501

Memorias del IX Encuentro Nacional de Experiencias en Enseñanza de la Biología y la

Educación Ambiental. IV Congreso Nacional de Investigación en Enseñanza de la Biología.

includes soliciting situations as well as educational spaces that motivate the argumentation. The recording of the information, as well as the extraction of the data, is carried out with audio and video recordings of some sessions as well as observations documented in the field diary. To carry out the characterization of the argumentation the study is based on the theory of Toulmin. Methodologically, it begins with a pilot test based on the socio-scientific context called "early sexual relations". The results show patterns of argumentation, usually incomplete, common that are not based on expected knowledge. The final reflections lead to consider that efforts should be made to design learning environments that raise the argument and therefore the decision making in context; In that sense the investigation will continue projected to the central case: "addiction to psychoactive substances"

KEYWORDS: Argumentation, socio - scientific case, psychoactive substances, decision making.

\section{INTRODUCCIÓN}

Los procesos argumentativos constituyen la base para el desarrollo del pensamiento crítico de los estudiantes, además de ser una de las competencias que evidencian el aprendizaje. En todos los ámbitos educativos, la argumentación crea una oportunidad para tomar parte de diversas situaciones que surgen dentro de las diferentes disciplinas.

Hacer ciencia implica discutir, razonar, argumentar, criticar y justificar ideas y explicaciones; además, aprender ciencias demanda destrezas relacionadas estrechamente con el lenguaje, por lo tanto, el aprendizaje se presume como proceso social que requiere una actividad discursiva importante. De acuerdo a ello, se reconoce una relación entre las competencias comunicativas y el aprendizaje de los modelos científicos, lo que conlleva a inferir que una mejora en estas competencias corresponde a un aprendizaje de mayor calidad; y que aprender a pensar pasa por aprender a argumentar (Henao\&Stipcich, 2008).

Otro elemento que se fortalece a partir de la argumentación, se acepta en la propuesta constructivista, el cual menciona que "las teorías cotidianas - con lenguaje cotidianopuedan coexistir con teorías más científicas - con lenguaje científico -, siempre que se delimiten perfectamente sus contextos de uso. Si esto fuese posible es en la exposición y contraste de éstas donde los alumnos aprenden a delimitar y mejorar sus puntos de vista y a argumentar críticamente" (Pozo \& Gómez Crespo, 2000; Pozo \& Rodrigo, 2001 citado por Campaner \& De Longhi, 2007).

En didáctica de las ciencias, recientemente, se destaca el estudio de las competencias argumentativas y la creación de estrategias para potenciar estas competencias; tal como lo señala Solbes, Ruíz \& Furio (2000), quienes hacen referencia a los informes PISA que relacionan este tipo de competencias con la interpretación de evidencias y conclusiones científicas. 


\section{Bio - grafía. Escritos sobre la Biología y su Enseñanza. ISSN 2027-1034}

Edición Extraordinaria. p.p. 492 - 501

Memorias del IX Encuentro Nacional de Experiencias en Enseñanza de la Biología y la

Educación Ambiental. IV Congreso Nacional de Investigación en Enseñanza de la Biología.

A través de la enseñanza de la biología se desarrollan temáticas que pueden enriquecer el contexto y la toma de decisiones dentro del mismo, por tanto considerados como asuntos socio - científicos, que permiten la participación activa de los estudiantes, la construcción de nuevos conocimientos y el uso de los mismos dentro de su contexto. El asunto a tratar dentro de la investigación: "adicción a sustancias psicoactivas", corresponde a una problemática actual, de interés y con diferentes posibilidades de discusión y resolución.

Es común que en el aula los estudiantes se les dificulten la manera de comunicar sus ideas o expresar apropiadamente su desacuerdo frente a la opinión de otra persona, lo que conduce a justificaciones y argumentos carentes de sentido, de conceptos e incluso pertinencia. Si bien la competencia argumentativa es en parte producto de una correcta interpretación, también hace parte de ésta la capacidad explicativa y discursiva; desde este punto de vista se considera pertinente identificar los esquemas argumentativos de los estudiantes con base en la propuesta de Toulmin (1958) e incentivar estrategias que permitan alcanzar en los estudiantes propuestas argumentativas sólidas y enriquecidas en cuanto a lenguaje y conceptos.

El desarrollo de este asunto se efectúa a partir de una unidad didáctica que se enriquece con la implementación de actividades como lecturas, consultas, organización de ideas, foros y exposiciones que permitan a los estudiantes presentar sus opiniones, reflexiones, objeciones y justificaciones; de esta manera se caracterizan los argumentos que se identifican a lo largo de las actividades y que permitan visibilizar la asociación con conocimientos adquiridos, la capacidad crítica, la posibilidad de toma de decisiones y la expresión de todo lo anterior, usando herramientas tecnológicas como los pod cast.

\section{METODOLOGÍA}

De acuerdo con el objetivo principal planteado en la investigación, el cual se centra en la identificación de esquemas argumentativos en estudiantes de grado $8^{\circ}$, a partir de una situación específica, este estudio se cataloga como un enfoque cualitativo, de acuerdo con la descripción que realiza Hernández, R; Fernández, C \& Baptista, M (2014)ya que "se enfoca en comprender los fenómenos, explorándolos desde la perspectiva de los participantes en un ambiente natural y en relación con su contexto".

El estudio se realiza en un grupo conformado por 22 estudiantes de grado $8^{\circ}$, de un Colegio público de la ciudad de Bogotá - Colombia, durante el año 2017. La edad de los estudiantes oscila entre los 12 y 17 años, aunque más del 50\% se encuentran entre 12 y 13 años, por tanto, son jóvenes que a nivel académico manifiestan interés y su participación es constante.

El asunto particular propuesto está coligado a las temáticas del plan de estudios, dado que en grado $8^{\circ}$, uno de los contenidos planteados desde el Ministerio de Educación Nacional, a través de los estándares curriculares corresponde al sistema nervioso y sus cuidados; teniendo en cuenta este contexto y las condiciones sociales dentro de las que se encuentra el grupo, se propone abordar esta temática que se representa dentro de su 
Bio - grafía. Escritos sobre la Biología y su Enseñanza. ISSN 2027-1034

Edición Extraordinaria. p.p. 492 - 501

Memorias del IX Encuentro Nacional de Experiencias en Enseñanza de la Biología y la Educación Ambiental. IV Congreso Nacional de Investigación en Enseñanza de la Biología.

cotidianidad y que genera discusión, por tanto diversos puntos de vista y posibilidad argumentativa.

El método fundamental para realizar la recolección de datos consiste en la observación, grabación de audio y registros en diario de campo, tal como se muestra en la tabla 1. Dadas estas condiciones, se implementó una prueba piloto en la cual se diseñó una actividad que promovió la actividad argumentativa, ésta consistió en el desarrollo de un foro en relación a la temática "Relaciones sexuales a temprana edad".

Tabla 1. Descripción fases del diseño metodológico de la investigación.

Fuente. Los autores.

\section{DISENO METODOLOGICO}

- Planteamiento de objetivos, Análisis de contexto, situaciones en el aula, construcción teórica, revisión bibliográfica

diligenciamiento de diario de campo para construcción de propósitos y revisión teórica.

- Diseño, ajuste y construcción de herramientas instrumentos didácticos

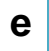

e

Construcción de herramientas e instrumentos que promuevan la actividad argumentativa:

- Actividad piloto

- Actividad de aplicación
- Aplicación de instrumentos y herramientas didácticas (recopilación de datos)

- Aplicación de instrumentos y herramientas (recopilación de datos)

- Análisis y evaluación de actividades

- Caracterización y evaluación de esquemas argumentativos - conclusiones
Aplicación de pilotaje a través de un foro con la temática "Relaciones sexuales a temprana edad".
Aplicación de secuencia didáctica

Juego de roles

Foro: "Uso de sustancias psicoactivas y adicción"

Se realizará el análisis de actividades aplicadas usando la matriz de relativización propuesta.

Se caracterizarán los esquemas argumentativos, basados en la teoría de Toulim (1958 y 2003). Para ello se usará la rejilla de caracterización 
Bio - grafía. Escritos sobre la Biología y su Enseñanza. ISSN 2027-1034

Edición Extraordinaria. p.p. 492 - 501

Memorias del IX Encuentro Nacional de Experiencias en Enseñanza de la Biología y la Educación Ambiental. IV Congreso Nacional de Investigación en Enseñanza de la Biología.

\begin{tabular}{|l|l|}
\hline & propuesta por Henao (2010), con algunos ajustes. \\
& $\begin{array}{l}\text { Se reportarán resultados, análisis y conclusiones } \\
\text { de acuerdo a los objetivos planteados. }\end{array}$ \\
\hline - Producción de podcast & $\begin{array}{l}\text { Los estudiantes llevarán sus argumentos a un } \\
\text { programa radial que será publicado como pod } \\
\text { cast. }\end{array}$ \\
\hline
\end{tabular}

El modelo argumentativo de Toulim (1958), expone categorías que determinan los elementos que se encuentran en los enunciados, de tal manera que se puedan caracterizar los argumentos que producen los estudiantes. Las categorías se discriminan de la siguiente manera:

- Dato

- Respaldo a dato

- Garantía

- Respaldo a garantía

- Cualificador modal

- Refutación

- Conclusión

A partir de las cualidades argumentativas, las categorías argumentativas y la relación de saberes con la producción de argumentos, se toma la rejilla propuesta por Henao (2010) y modificada por Mesa \& Seña (2013) para analizar y comprender los elementos del modelo argumentativo en torno a los argumentos que surgen de los estudiantes, y como estos se relacionan de manera coherente o no, con el asunto propuesto en la discusión. 
Bio - grafía. Escritos sobre la Biología y su Enseñanza. ISSN 2027-1034

Edición Extraordinaria. p.p. 492 - 501

Memorias del IX Encuentro Nacional de Experiencias en Enseñanza de la Biología y la

Educación Ambiental. IV Congreso Nacional de Investigación en Enseñanza de la Biología.

Tabla 2. Rejilla para identificar características en los argumentos de los estudiantes.

Fuente. Adaptada de Mesa \& Seña (2013).

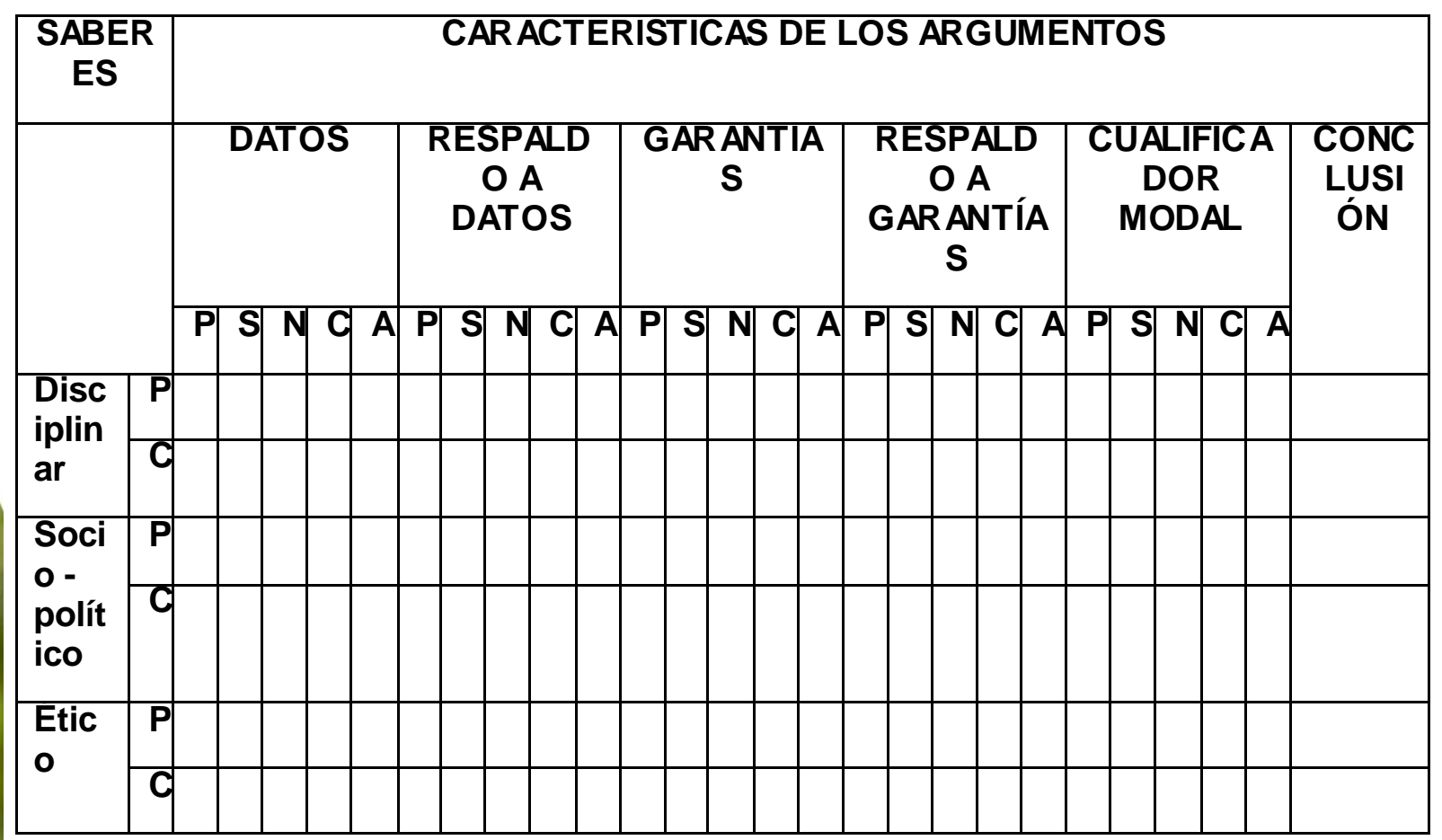

La rejilla representada en la tabla 2, se visualiza con cada estudiante en la prueba piloto; en ese sentido se presentan los resultados obtenidos hasta la fecha.

\section{RESULTADOS Y DISCUSIÓN}

A continuación se presentan los resultados analizados hasta la fecha, con base en la prueba piloto aplicada en el mes de abril de 2017. La producción de argumentos en la prueba piloto se realiza a partir de un texto informativo en el que se expone una noticia, desde esta se propone una situación problema y distintas soluciones de manera implícita.

Durante el debate desarrollado en la prueba piloto, se despliegan distintas discusiones en torno a la lectura. Una de estas discusiones correspondía al embarazo adolescente y las causas del mismo, a partir de esta discusión se extraen fragmentos argumentativos y se caracterizan como se observa en la figura 1. 
Bio - grafía. Escritos sobre la Biología y su Enseñanza. ISSN 2027-1034

Edición Extraordinaria. p.p. 492 - 501

Memorias del IX Encuentro Nacional de Experiencias en Enseñanza de la Biología y la Educación Ambiental. IV Congreso Nacional de Investigación en Enseñanza de la Biología.

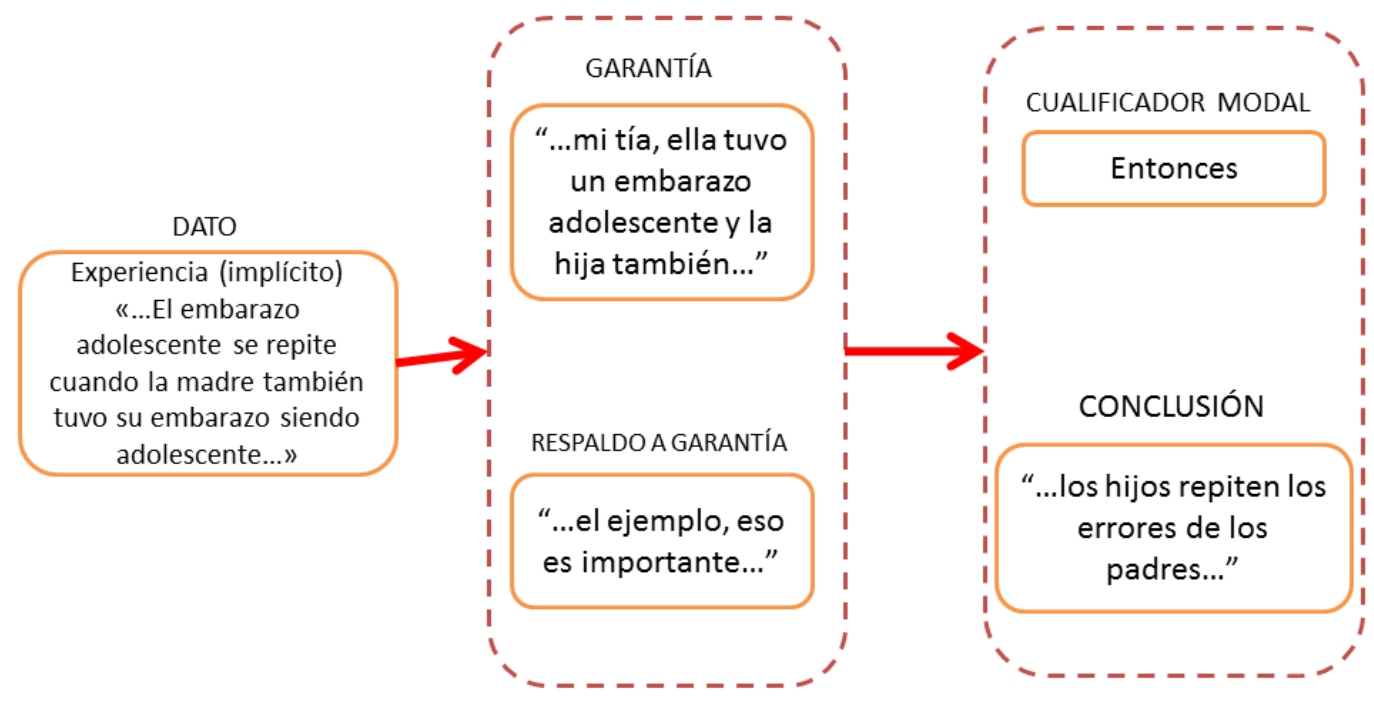

Figura 1. Argumento de estudiante 1 (E1).

Fuente. Los autores.

El estudiante 1 (figura 1), particularmente, presenta un tipo de justificación empírica según la categorización de justificaciones que realiza Kelly et. al (1998 - Citado por: López \& Jiménez, 2007), y a pesar de que la estructura contiene los elementos básicos argumentativos, estos no presentan suficiencia para lograr persuadir a sus compañeros; algunos de ellos generan nuevos argumentos como el que se extrae en la figura 2.

Los argumentos analizados presentan un componente en común: ninguno se basa en saberes disciplinares para ofrecer un argumento; los estudiantes toman sus vivencias sin contemplar saberes adquiridos en el colegio, tal como se observa en la figura 2.

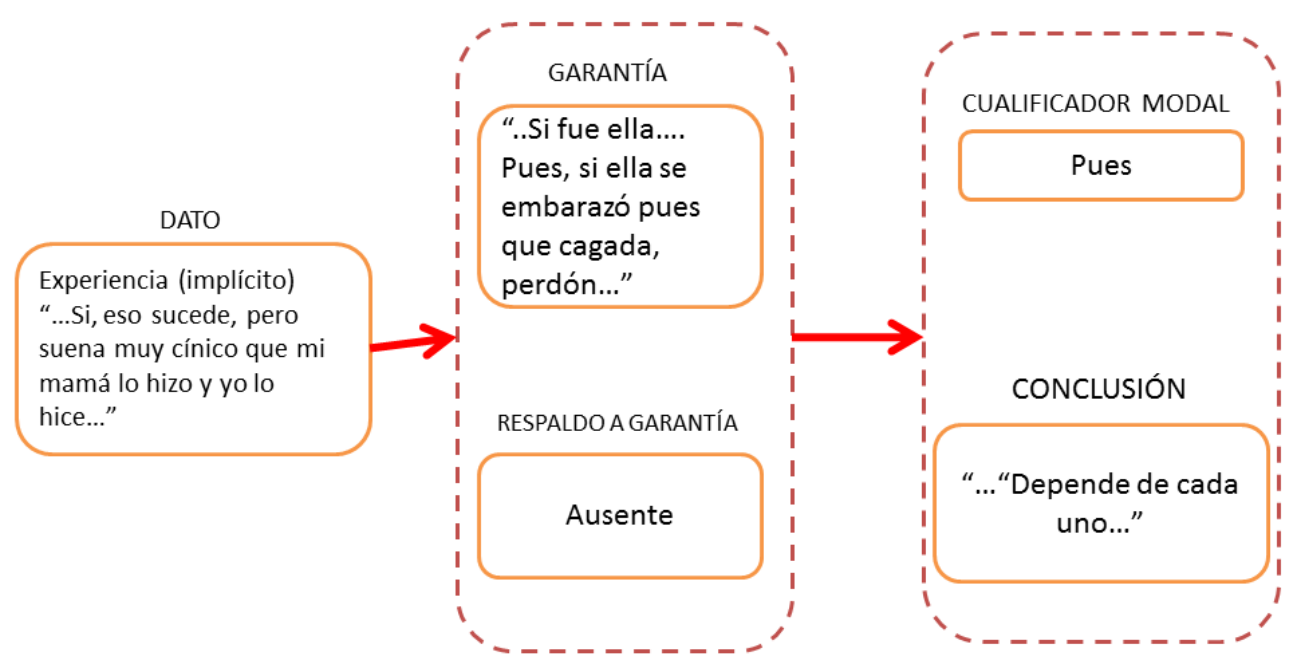


Bio - grafía. Escritos sobre la Biología y su Enseñanza. ISSN 2027-1034

Edición Extraordinaria. p.p. 492 - 501

Memorias del IX Encuentro Nacional de Experiencias en Enseñanza de la Biología y la Educación Ambiental. IV Congreso Nacional de Investigación en Enseñanza de la Biología.

Figura 2. Argumento Estudiante 2 (E2)

Fuente. Los autores.

En otro momento del debate se discute acerca de la responsabilidad de los padres respecto al inicio de una vida sexual temprana. De acuerdo a ello se caracteriza uno de los argumentos, mostrado en la figura 3, durante esta situación.

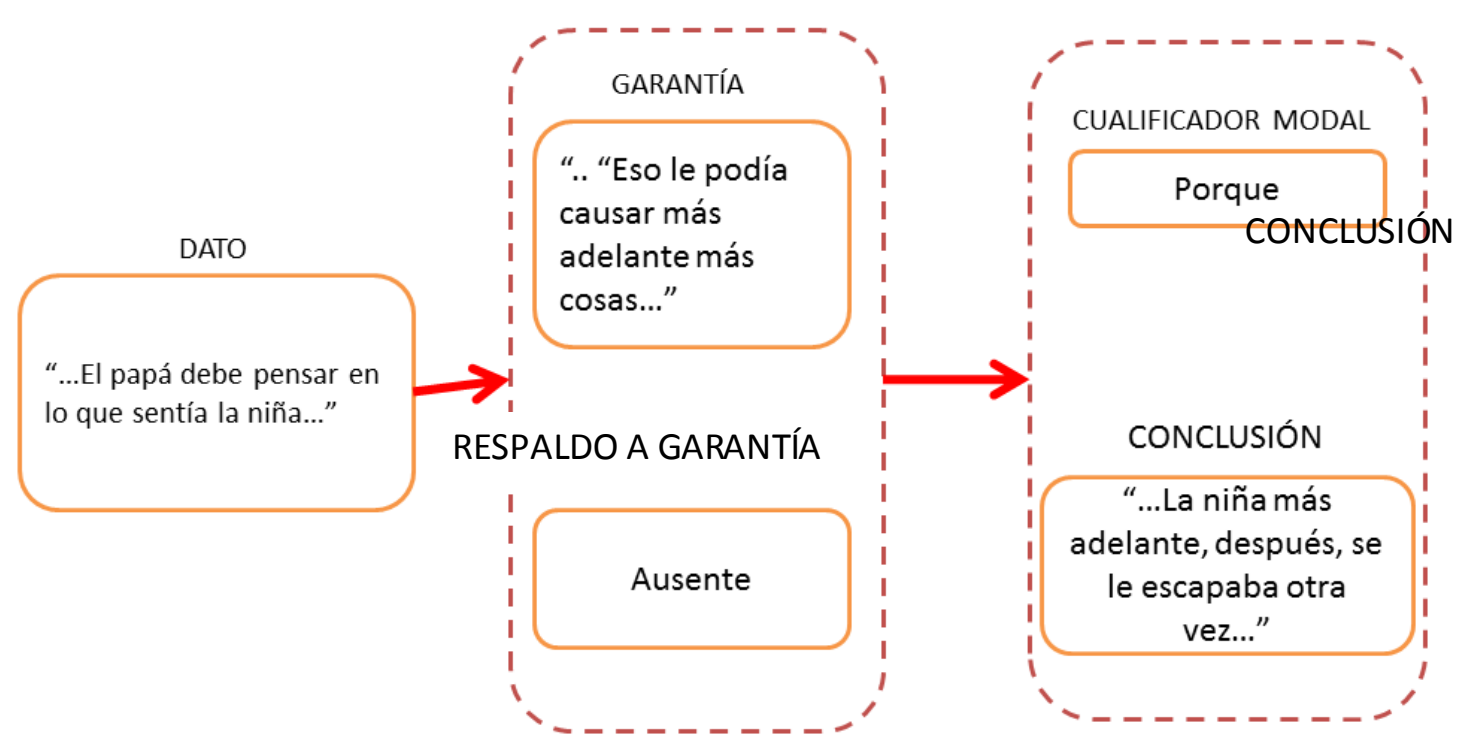

Figura 3. Argumento Estudiante 3 (E3)

Fuente. Los autores.

Tanto el argumento del estudiante 2 como el del estudiante 3 carecen de respaldo a su justificación, por lo que se puede evidenciar la baja calidad en los argumentos, de acuerdo con la afirmación realizada por López \&Jiménez en 2007, quienes consideran de mejor calidad los argumentos justificados, es decir los que presentan evidencias (garantía y respaldo a garantía).

Concordando con el estudio de Solbes, Ruíz y Furió (2010) se observó cualitativamente la influencia de aspectos afectivos y sociales en los debates, en este caso, los estudiantes recurrieron a experiencias propias o cercanas a su contexto; sin embargo, en ocasiones fue necesario intervenir para persuadir el debate y obtener mayor participación.

Un punto importante dentro del análisis corresponde al escaso uso de saberes disciplinares, lo cual conlleva a determinar que no se cumplirán, o se cumplirán parcialmente los objetivos de aprendizaje, puesto que (Kelly \& Takao, 2002; citado por: López \& Jiménez 2007), consideran que la argumentación es un elemento útil para explorar el desarrollo de la comprensión conceptual. Además de ello, López \& Jiménez 
Bio - grafía. Escritos sobre la Biología y su Enseñanza. ISSN 2027-1034

Edición Extraordinaria. p.p. 492 - 501

Memorias del IX Encuentro Nacional de Experiencias en Enseñanza de la Biología y la

Educación Ambiental. IV Congreso Nacional de Investigación en Enseñanza de la Biología.

(2007), indican la relevancia al relacionar los enunciados teóricos y los datos o justificaciones para explorar la apropiación del discurso científico por parte del alumnado.

\section{CONCLUSIONES}

Si bien, esta es una investigación en desarrollo, se puede inferir que es necesario implementar distintas actividades que refuercen y estructuren los saberes disciplinares, como base para la adquisición de elementos argumentativos.

Las situaciones elicitadoras son fundamentales en la construcción de secuencias didácticas que motiven y traten temáticas 0 asuntos socio-científicos en contexto, actividades que aportan en la construcción de argumentos.

A la altura de desarrollo de la investigación, en los casos analizados, se identifica que es preciso incrementar el nivel de argumentación, así mismo se requiere que estos argumentos consideren conocimientos científicos.

La carencia de elementos disciplinares en los argumentos de los estudiantes señala que éstos prefieren usar sus experiencias al momento de justificar situaciones como las propuestas en la investigación. Lo anterior podría obedecer a que a los estudiantes se les dificulta asociar los conceptos disciplinares a su cotidianidad. Desde otra perspectiva, los estudiantes optan por utilizar las experiencias, que reconocen como significativas para su contexto, para construir los argumentos en medio de una situación que implique este tipo de interacción. Este último aspecto permite replantear la metodología e incluir el diseño de una secuencia didáctica que apoye la adquisición de herramientas conceptuales significativas en el proceso argumentativo.

\section{BIBLIOGRAFÍA}

Campaner, Gertrudis \& De Longhi, Ana. (2007). La argumentación en educación ambiental. una estrategia didáctica para la escuela media. En: Revista electrónica de Enseñanza de las ciencias Vol. 6, №2 pp $442-456$.

Henao, Berta \&Stipcich, María. (2008). Educación en ciencias y argumentación: la perspectiva de Toulim como posible respuesta a las demandas y desafíos contemporáneos para la enseñanza de las ciencias experimentales. En: Revista electrónica Enseñanza de la ciencias, vol 7, №1 pp 47 - 62.

Henao, Berta; Stipcich, María\& Moreira, María. (2010). Una perspectiva epistemológica como nicho y posibilidad para propiciar la enseñanza como argumentación. II Congreso Internacional de Didactiques. 
Bio - grafía. Escritos sobre la Biología y su Enseñanza. ISSN 2027-1034

Edición Extraordinaria. p.p. 492 - 501

Memorias del IX Encuentro Nacional de Experiencias en Enseñanza de la Biología y la Educación Ambiental. IV Congreso Nacional de Investigación en Enseñanza de la Biología.

Hernández, Roberto; Fernández, Carlos \& Baptista, Pilar (2014). Metodología de la investigación. México. Editorial Mc Graw Hill.

López, Ramón \& Jiménez, María (2007). ¿Podemos cazar ranas? calidad de los argumentos de alumnado de primaria y desempeño cognitivo en el estudio de una charca. En: Revista Enseñanza de las Ciencias, Vol 25 №3, pp 309 - 324.

Mesa, Sandra \& Seña, Erika (2013). Argumentación en torno al concepto "lo vivo": discusiones sobre el maltrato animal como asunto socio científico. Universidad de Antioquia.

Solbes, Jordi; Ruíz, Juan \&Furio, Carles (2000) Debates y argumentación en las clases de física y química; En: Alambique Didáctica de las ciencias experimentales. № 63 p $65-75$.

Toulim, Stephen (2003). Regreso a la razón. Barcelona. Ediciones Península.

Toulmin, Stephen (1958 y 2003). Los usos de la argumentación. Barcelona. Ediciones Península. 